Check for updates

Cite this: RSC Adv., 2019, 9, 958

Received 8th October 2018

Accepted 24th December 2018

DOI: $10.1039 / \mathrm{c} 8 \mathrm{ra0} 0323 \mathrm{a}$

rsc.li/rsc-advances

\section{Superhydrophilic fluorinated polyarylate membranes via in situ photocopolymerization and microphase separation for efficient separation of oil-in-water emulsion $\uparrow$}

\author{
Hui Li, (D)*ab Cuiping Zhou, ${ }^{a}$ Chunsheng Li, ${ }^{a}$ Xiaohui Li ${ }^{a}$ and Shuxiang Zhang*b \\ A superhydrophilic modified fluorinated polyarylate membrane with high tensile strength was prepared by \\ a combination of in situ photocopolymerization and microphase separation. The as-prepared membrane \\ was successfully utilized for oil-in-water emulsion separation with high separation efficiency and high \\ flux. Furthermore, the membrane displayed excellent antifouling performance and recyclability for long- \\ term use.
}

Today, the ever-growing serious environmental pollution caused by oil-contaminated water from the daily life of people as well as from industries demands the search for novel materials and strategies to realize oil/water separation with high efficiency. ${ }^{1-5}$ Traditional separation technologies such as gravity separation, centrifugation, skimming, sedimentation, and flotation are useful for most of the separation processes. Unfortunately, low separation efficiency, high energy consumption and complex equipment have restricted the application of these technologies to some extent..$^{6-8}$ Other than that, it may be very difficult for them to separate emulsified oil/water solutions. ${ }^{9}$ Therefore, desirable materials for effective separation of oil/water emulsions are urgently needed. As a result, filtration polymer membranes have been considered to be a suitable technology for separating various emulsions, but suffer from low flux, surface fouling and poor mechanical properties., ${ }^{2,9}$

Recently, significant interests have been attracted to the design and preparation of oil/water separation membranes with special wettability by a combination of rough structure and surface chemistry. ${ }^{2,10-14}$ Typically, these polymer membranes may be classed into two types, polymer coated mesh membranes and polymer porous membranes. ${ }^{3,15-22}$ For polymer coated mesh membrane, it requires a mesh as a support which is capable of improving mechanical properties and rendering

\footnotetext{
${ }^{a}$ School of Chemistry and Chemical Engineering, University of Jinan, Jinan 250022, China.E-mail: chm_lih1@ujn.edu.cn

${ }^{b}$ Shandong Key Laboratory of Fluorine Chemistry and Chemical Engineering Materials, University of Jinan, Jinan 250022, China. E-mail: chm_zhangsx@ujn.edu.cn

$\dagger$ Electronic supplementary information (ESI) available: Experimental section, characterization methods, synthetic route and GPC curve of FPAR, the diagram of the SFPAR membrane, droplet size distribution of the emulsion, UV-VIS spectra for the toluene-in-water emulsion before and after filtration, stress-strain curve of the FPAR and SFPAR membranes, oil purity of the filtrate separated from the oil-in-water emulsions. See DOI: 10.1039/c8ra08323a
}

a micro-scale porous structure. ${ }^{2}$ For example, Tuteja and coworkers developed a superhydrophobic mesh membrane coated with a blend of cross-linked poly(ethylene glycol)diacrylate and fluorodecyl polyhedral oligomeric silsesquioxane, which was valuable for separation of oil/water emulsions with droplet sizes larger than $1 \mu \mathrm{m} .{ }^{21} \mathrm{PVDF}$ has been acknowledged as one of the main materials for manufacturing polymer porous membranes for separation of oil/water emulsions through a phase-inversion process. ${ }^{1,2}$ In 2014 , a superhydrophilic and underwater superoleophobic poly-(acrylic acid)-grafted PVDF (PAA- $g$-PVDF) membrane was fabricated by a salt-induced phase-inversion approach and applied to oil-in-water emulsions, however, the tensile strength of this membrane was not more than $0.64 \mathrm{MPa}$, which limited their practical applications. ${ }^{22}$

Polyarylate, a family of high-performance polymers, noted for their strength, toughness, chemical resistance, and high melting points. ${ }^{23-26}$ Recently, Livingston and co-workers have demonstrated the formation of crosslinked polyarylate microporous membranes which have great potential for applications in molecular separations. ${ }^{27}$ In previous studies, our group developed a simple procedure to fabricate a superhydrophobic and superoleophilic porous polyarylate membrane which could effectively separate oil/water mixtures. ${ }^{28}$ In this communication, we reported the fabrication of a novel superhydrophilic sodium acrylate modified fluorinated polyarylate (SFPAR) membrane for efficient separation of oil-in-water emulsion by a combination of in situ photocopolymerization and microphase separation. It was very exciting that the as-prepared SFPAR membrane exhibited prominent mechanical strength and outstanding water permeability. Furthermore, the membrane also displayed excellent underwater superoleophobicity, antifouling performance and recyclability for long-term use, which highlight its potential for practical applications. 
Fig. 1 shows the formation of a SFPAR membrane via in situ photocopolymerization for endowing with the hydrophilic property of FPAR (Scheme 1a-c), followed a microphase separation (Scheme 1d and e) for obtaining the SFPAR membrane with porous structure. The experiments are described in detail in the ESI. $\dagger$ Here, in situ photocopolymerization was applied for getting hydrophilic FPAR, which have the following advantages: good dispersibility of the formed acrylate copolymer in the FPAR matrix, low reaction temperature, and shortening the preparation time of membrane. After a microphase separation and a drying process, a white membrane was obtained by peeling from a substrate (Scheme 1f).

One of the main purposes of in situ photocopolymerization is to improve the wettability of FPAR by introducing carboxylate salts (Fig. S1b $\dagger$ ). Fig. 1a shows water contact angle of the FPAR membranes as function of sodium acrylate mass fraction. The results indicated that the value of water contact angle on the FPAR surface significant decreased with the increase of the sodium acrylate content. After the sodium acrylate content exceeded $\sim 9.5 \mathrm{wt} \%$, the contact angle tended to equilibrium, less than $\sim 1^{\circ}$, which formed a superhydrophilic modified FPAR membrane (SFPAR). To further examine the wettability of water on the FPAR membranes, the water contact angles of the FPAR and SFPAR membrane as function of time was also measured (Fig. 1b). The pure FPAR membrane had the initial water contact angel of approximately $96.2^{\circ}$ and the value of water contact angel almost kept stable after $100 \mathrm{~s}$, exhibiting good hydrophobicity. On the contrary, the approach to introducing carboxylate to FPAR caused a significant differences. The SFPAR membrane had the initial water contact angel of approximately

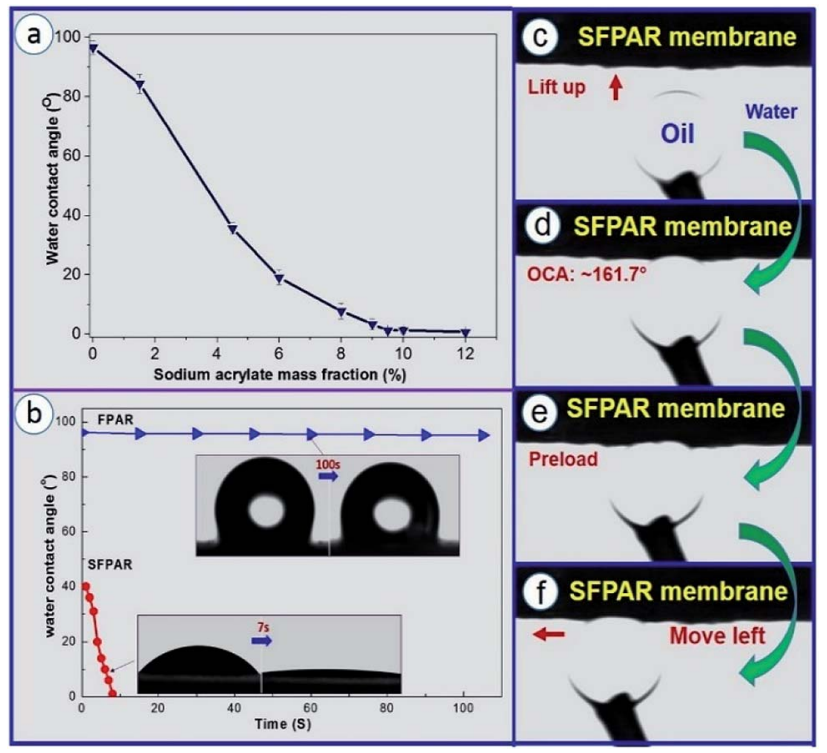

Fig. 1 Water contact angle of the FPAR membranes as function of sodium acrylate mass fraction (a), water contact angle of the SFPAR and FPAR membranes as function of time (the insets are photographs of water drops on the membrane surfaces) (b), underwater-oil contact angle ( $c$ and $d$ ) and dynamic underwater-oil-adhesion of the SFPAR membrane (e and f). The underwater-oil contact angle were measured with $4 \mu \mathrm{L}$ hexadecane droplet.

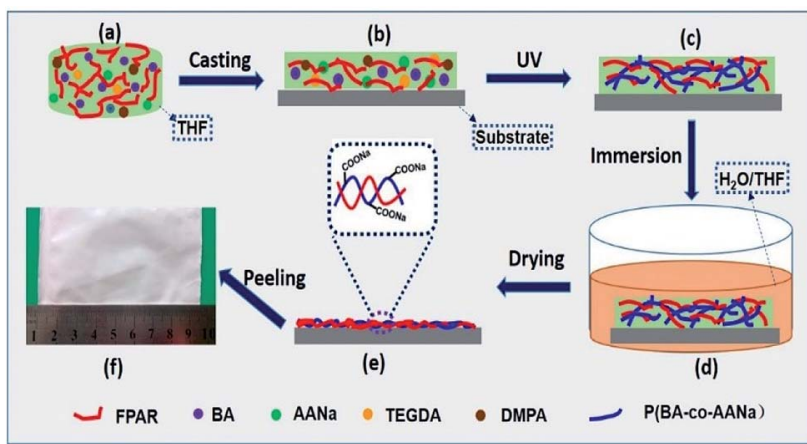

Scheme 1 Schematic of the formation of a SFPAR membrane via in situ photocopolymerization and a limited micro-phase separation. The fluorinated polyarylate (FPAR) was fabricated by interfacial polymerization of bisphenol AF, terephthaloyl chloride, and isophthaloyl chloride (Fig. S1a†). The number-average molecular weight of the obtained PAR is 93000 and the polydispersity index is 1.76 (Fig. S2†). The experiments are described in detail in the ESI. $\dagger$

$40^{\circ}$. Interestingly, the value of water contact angel of the SFPAR membrane rapidly decreased to $\sim 1^{\circ}$ in less than $7 \mathrm{~s}$, illustrating outstanding superhydrophilicity, which is caused by the introduction of carboxylate sodium and the porous structure of the SFPAR membrane. Furthermore, the underwater-oil contact angle (OCA) and dynamic underwater-oil-adhesion of the SPAR membrane were also studied (Fig. 1c and d). An oil droplet was lifted up and contacted the SPAR membrane surface under water (Fig. 1c and d). It was observed that the oil droplet remained spherical and the underwater-OCA of this membrane is $\sim 161.7^{\circ}$, demonstrating excellent underwater superoleophobicity. From Fig. 1e to Fig. 1f, the oil droplet was forced to adequately contact the membrance surface and then moved to the left. During the moving process, the spherical oil droplet had no obvious deformation, also showing that the SPAR membrane had excellent antiadhesion to oil.

ATR-FTIR spectra of the SFPAR and FPAR membranes are shown in Fig. 2a. For ATR-FTIR spectrum of the SFPAR membrane, besides the corresponding absorption peak of FPAR, The characteristic stretching peaks were obviously shown at $2850-3000 \mathrm{~cm}^{-1}$ and $1457 \mathrm{~cm}^{-1}$, respectively, resulting from $-\mathrm{CH}_{2}-$ and $-\mathrm{CH}_{3}$, and $-\mathrm{O}-\mathrm{CH}_{2}-$ groups of the crosslinked acrylate copolymer prepared by in situ photocopolymerization. The peak at $1569 \mathrm{~cm}^{-1}$ was the asymmetric $\mathrm{CO}^{2-}$ (salts) stretching vibration in $-\mathrm{CO}_{2} \mathrm{Na}$ of the formed acrylate copolymer. Moreover, the peak at $1640 \mathrm{~cm}^{-1}$, which was attributed to the stretching vibration of vinyl bond, was not observed from the ATR-FTIR spectrum of the SFPAR membrane, indicating that the monomers were polymerized.

XPS was performed to examine the surface chemical composition. Fig. 2b exhibits the overall XPS spectra of the SFPAR and FPAR membranes. There were three signals on the surface of the FPAR membrane attributed to $\mathrm{C}, \mathrm{O}$ and $\mathrm{F}$ element whose atomic percentage was approximately 66.0, 20.6, and $13.4 \%$, respectively. In comparison with the XPS spectrum of FPAR, the new signal appearing in the spectrum of the SFPAR membrane was attributed to Na element. The percentage of $\mathrm{Na}$ was estimated to be approximately $4.5 \mathrm{wt} \%$, higher than the 

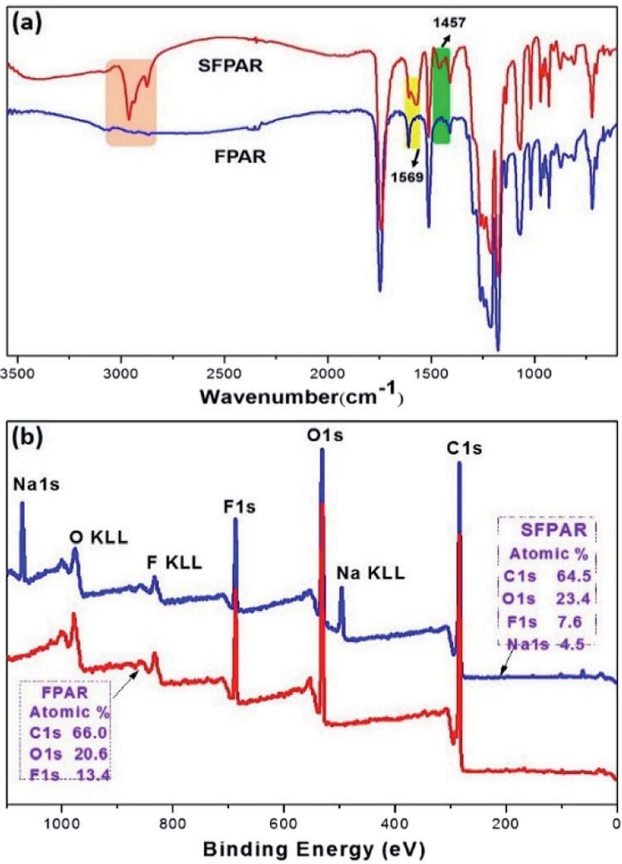

Fig. 2 ATR-FTTR spectra of the SFPAR and FPAR membranes (a) and the overall XPS spectra of the SFPAR and FPAR membranes (b).

bulk content ( $2.3 \mathrm{wt} \%$ ), and the F content of SFPAR membrane was obvious decreased and the $\mathrm{O}$ content is increased after in situ photocopolymerization, indicating the obvious surface enrichment of sodium carboxylate groups in the SFPAR membrane.

Fig. 3 displays SEM images of the surface and cross section of the FPAR and SFPAR membranes. Apparently, the morphologies of the SFPAR membrane are different from those of the FPAR which can be attributed to the thermodynamics instability and the non-solvent Induce phase separation. The FPAR surface
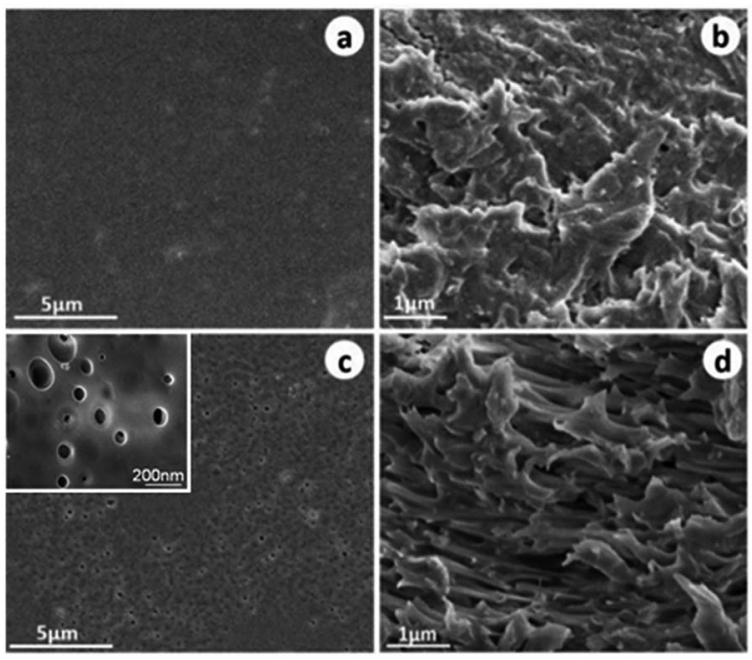

Fig. 3 SEM images of the FPAR and SFPAR membranes: the surface (a) and cross section (b) of the FPAR membrane; the surface (c) and cross section (d) of the SFPAR membrane. The inset is high-magnification SEM image of the SFPAR membrane surface. is smooth (Fig. 3a), while the SFPAP surface is porous and has a great number of micro nano-scale pores (Fig. $3 \mathrm{c}$ ) and the pore size and pore distribution were calculated by Nano Measurer 1.2 (Fig. S3a †). For the cross of the FPAR and SFPAR membranes, the former is dense and few pores can be found (Fig. 3b). However, the latter is loose and possesses many inter-connected nano-scale channels with a diameter of 50-200 nm (Fig. 3d). Different from the reported superhydrophilic membranes made from semicrystalline PVDF, ${ }^{22,29,30}$ the FPAR is amorphous. According to the XPS and SEM results above, the possible formation mechanism of SFPAR membranes with porous structure prepared by in situ photocopolymerization and phase separation can be described as follows. As can be seen from Scheme 1, the FPAR, the monomers (BA, SA and TEGDA) and photoinitiator are first dissolved in THF to form the homogenous viscous solution (Scheme 1a and b). After in situ photocopolymerization of $\mathrm{BA}, \mathrm{SA}$ and TEGDA occurs at room temperature, the crosslinked polyacrylate containing sodium carboxylate groups come into being in the viscous solution (Scheme 1c). During the immersion process (Scheme 1d), with the extraction of THF by the coagulation bath, the blend matrix of the amorphous FPAR and the crosslinked polyacrylate will gradually shrink and solidify. Simultaneously, a microphase separation occurs in the blend matrix due to the crosslinking of polyacrylate and the thermodynamics instability. Furthermore, sodium carboxylate groups attached to the polyacrylate network can absorb enough water in the blend matrix, ultimately leading to the formation of the wet membrane containing water. During the drying process, water is evaporated from the wet membrane and the porous structure appears in the membrane because the solidification of FPAR matrix restricts the movement of the polyacrylate segments. Finally, the SFPAR membrane with porous structure is obtained after the blend matrix is fully dried at room temperature (Scheme 1e and f). The hydrophilic sodium carboxylate groups will enrich in the SFPAR membrane surface and the inner surface of the micro nano channel due to the driving forces of surface free energy and hydrophilicity/ hydrophobicity interactions (Fig. S3b, ESI $\dagger$ ), which makes it possible for the preparation of oil-water separation membrane.

In this work, oil-water separation of the SFPAR membrane was carried out with a vacuum driven filtration system at 0.07 MPa. Toluene-in-water emulsion was employed to evaluate the separation ability of the membrane and the droplet size distribution of the emulsion is in the range from $\sim 900 \mathrm{~nm}$ to $\sim 8 \mu \mathrm{m}$ (Fig. S4, ESI $\dagger$ ). Fig. 4a illustrates a self-made separation device and the separation result of toluene-in-water emulsion (the separation experiments are described in detail in the ESI + ). Compared with the milky white feed emulsion (up), the filtrate (down) is colorless from the appearance. A noticable difference was observed between the feed and the filtrate by the optical microscopy images. There appear a great many droplets in the image of the feed before filtration, however, no droplet can be viewed for the filtrate. Furthermore, the characteristic peak of toluene for the filtrate is not observed from UV-VIS spectrometer (TU-1901, Beijing Purkinje General Instrument Co., Ltd, China) in comparison with the feed (Fig. S5, ESI†), and the oil content in the filtrate is $54 \pm 17 \mathrm{ppm}$ measured by a total 


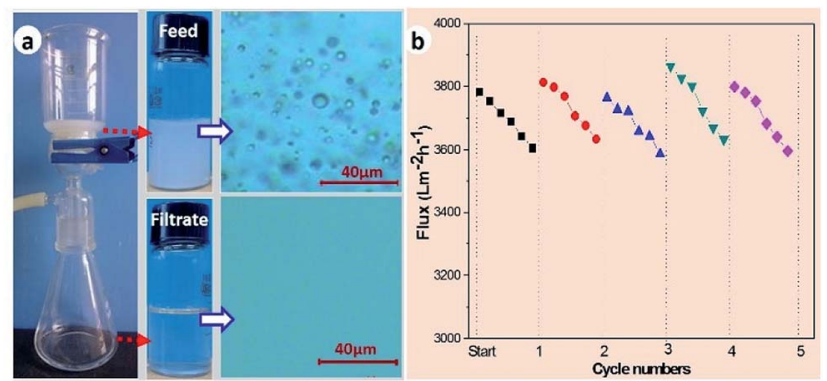

Fig. 4 The vacuum driven filtration system and separation results for toluene-in-water emulsion (a) and change of the flux and flux recovery in the separation of a toluene-in-water emulsion over five cycles (b).

organic carbon analyzer, indicating that the as-prepared membrane can successfully separate oil/water emulsion with high efficiency. The other two emulsions also have good separation efficiency (Table $\mathrm{S} 1, \mathrm{ESI} \dagger$ ).

Taking advantage of the reported method, ${ }^{22,29}$ the flux and the antifouling property of the membrane were measured by the vacuum driven filtration system. Continuous separation of the toluene-in-water emulsion lasts for approximately 30 hours over five cycles and the flux is detected every an hour and six points were taken down within each cycle. The SFPAR membrane is gently washed by using DI water to dispose of surface adsorbent. As shown in Fig. 4b, the flux has a slight decline from $\sim 3800$ to $\sim 3600 \mathrm{~L} \mathrm{~m}^{-2} \mathrm{~h}^{-1}$ within one cycle. Nevertheless, the membrane can recover fully to the initial flux after it is washed by water. The results show that the SFPAR membrane possesses a high flux and an outstanding antifouling performance for long-term use. Further studies will focus on the regulation of the pore size of the SFPAR membrane and get the most proper selectivity and penetration. Moreover, as one of the important factors in practical application, the tensile strength of the membrane was also tested by a testing machine (Fig. S6, ESI $\dagger$ ). Due to the porous structure, the tensile strength of the SFPAR membrane is $\sim 6.02 \mathrm{MPa}$, less than that of the FPAR membrane ( 27.59 MPa). However, the SFPAR membrane still has high mechanical property compared with the reported hydrophilic modified PVDF oil/water separation membrane. ${ }^{\mathbf{1 4 , 1 7}}$

In conclusion, we have developed a novel superhydrophilic modified FPAR membrane with porous structure by in situ photocopolymerization of acrylate monomers and subsequent microphase separation. The results of ATR-FTIR and XPS demonstrated that sodium carboxylate groups was immobilized in the FPAR membrane by in situ photocopolymerization. When the sodium acrylate content was beyond $\sim 9.5 \mathrm{wt} \%$, the asprepared SFPAR membrane exhibited prominent superhydrophilicity, underwater superoleophobicity, and water permeability. The SFPAR membrane could effectively separate oil-in-water emulsions with high separation efficiency and high flux. Significantly, the obtained membrane possessed a good antifouling property and could be recycled for long-time use. From a practical perspective, the SFPAR membrane had a higher mechanical strength than traditional hydrophilic polymeric membranes with similar permeation properties. Therefore, we anticipate that our membrane will have high potential in practical application for treating wastewater from the daily life and industries.

\section{Conflicts of interest}

There are no conflicts to declare.

\section{Acknowledgements}

The authors thank the Natural Science Foundation of Shandong Province (ZR2016EMM04, ZR2017ZC0529, 2015ZDXX0702A01), and the National Natural Science Foundation of China (51103061).

\section{Notes and references}

1 G. M. Geise, H. Lee, D. J. Miller, B. D. Freeman, J. E. Mcgrath and D. R. Paul, J. Polym. Sci., Part B: Polym. Phys., 2010, 48, 1685-1718.

2 Z. Xue, Y. Cao, N. Liu, L. Feng and L. Jiang, J. Mater. Chem. A, 2014, 2, 2445-2460.

3 W. Qing, X. Shi, Y. Deng, W. Zhang, J. Wang and C. Tang, J. Membr. Sci., 2017, 540, 354-361.

4 P. Calcagnile, D. Fragouli, I. S. Bayer, G. C. Anyfantis, L. Martiradonna, P. D. Cozzoli, R. Cingolani and A. Athanassiou, ACS Nano, 2012, 6, 5413-5419.

5 P. Thanikaivelan, N. T. Narayanan, B. K. Pradhan and P. M. Ajayan, Sci. Rep., 2012, 2, 230.

6 M. A. Gondal, M. S. Sadullah, M. A. Dastageer, G. H. McKinley, D. Panchanathan and K. K. Varanasi, ACS Appl. Mater. Interfaces, 2014, 6, 13422-13429.

7 Z. Chu, Y. Feng and S. Seeger, Angew. Chem., Int. Ed., 2015, 54, 2328-2338.

8 P. Chen and Z. Xu, Sci. Rep., 2013, 3, 2776-2781.

9 W. Zhang, Z. Shi, F. Zhang, X. Liu, J. Jin and L. Jiang, Adv. Mater., 2013, 25, 2071-2076.

10 G. Kwon, A. K. Kota, Y. Li, A. Sohani, J. M. Mabry and A. Tuteja, Adv. Mater., 2012, 24, 3666-3671.

11 Z. Wang, X. Jiang, X. Cheng, C. H. Lau and L. Shao, ACS Appl. Mater. Interfaces, 2015, 7, 9534-9545.

12 J. Zhang and S. Seeger, Adv. Funct. Mater., 2011, 21, 46994704.

13 Q. Ma, H. Cheng, A. Fane, R. Wang and H. Zhang, Small, 2016, 12, 2186-2202.

14 B. Wang, W. Liang, Z. Guo and W. Liu, Chem. Soc. Rev., 2015, 44, 336-361.

15 Z. Xue, A. Wang, L. Lin, L. Chen, M. Liu, L. Feng and L. Jiang, Adv. Mater., 2011, 23, 4270-4273.

16 A. Matin, U. Baig, M. A. Gondal, S. Akhtar and S. M. Zubair, J. Membr. Sci., 2018, 548, 390-397.

17 C. Gao, Z. Sun, K. Li, Y. Chen, Y. Cao, S. Zhang and L. Feng, Energy Environ. Sci., 2013, 6, 1147-1151.

18 J. Li, Y. Zhou and Z. Luo, Chem. Eng. J., 2017, 322, 693-701.

19 Y. Cai, D. Chen, N. Li, Q. Xu, H. Li, J. He and J. Lu, J. Membr. Sci., 2017, 543, 10-17.

20 Y. Shang, Y. Si, A. Raza, L. Yang, X. Mao, B. Ding and J. Yu, Nanoscale, 2012, 4, 7847-7854. 
21 A. K. Kota, G. Kwon, W. Choi, J. M. Mabry and A. Tuteja, Nat. Commun., 2012, 3, 1025-1032.

22 W. Zhang, Y. Zhu, X. Liu, D. Wang, J. Li, L. Jiang and J. Jin, Angew. Chem., 2014, 126, 875-879.

23 M. S. Mccaig and D. R. Paul, Polymer, 2000, 41, 629-637.

24 L. Tan and B. Tan, Chem. Soc. Rev., 2017, 46, 3322-3356.

25 L. Zeng, R. Li, P. Chen, J. Xu and P. Liu, J. Appl. Polym. Sci., 2016, 133, 43800.

26 L. Zhu, C. Wang, X. Mao, Z. Huang and S. Sheng, Polymer, 2016, 29, 1090-1096.
27 M. F. Jimenez-Solomon, Q. Song, K. E. Jelfs, M. MunozIbanez and A. G. Livingston, Nat. Mater., 2016, 15, 760-767.

28 H. Li, X. Zhao, P. Wu, S. Zhang and B. Geng, J. Mater. Sci., 2016, 51, 3211-3218.

29 M. Tao, L. Xue, F. Liu and L. Jiang, Adv. Mater., 2014, 26, 2943-2948.

30 M. Tao, F. Liu and L. Xue, J. Mater. Chem., 2012, 22, 91319137. 